Itinéraires Itinéraires

Littérature, textes, cultures

\title{
Poésies numériques tactiles : toucher les signes par la « main de l'œil », manipuler la matière à l'écran
}

Digital Tactile Poetry: To Touch Signs through the "Eye's Hand", to Manipulate the Substance on the Touch Screen

\section{Emmanuelle Pelard}

\section{OpenEdition}

\section{Journals}

Édition électronique

URL : http://journals.openedition.org/itineraires/3983

DOI : $10.4000 /$ itineraires.3983

ISSN : 2427-920X

\section{Éditeur}

Pléiade

\section{Référence électronique}

Emmanuelle Pelard, «Poésies numériques tactiles : toucher les signes par la « main de l'œil », manipuler la matière à l'écran », Itinéraires [En ligne], 2017-3 | 2018, mis en ligne le 15 juin 2018, consulté le 10 décembre 2020. URL : http://journals.openedition.org/itineraires/3983 ; DOI : https:// doi.org/10.4000/itineraires.3983

Ce document a été généré automatiquement le 10 décembre 2020.

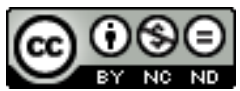

Itinéraires est mis à disposition selon les termes de la licence Creative Commons Attribution - Pas d'Utilisation Commerciale - Pas de Modification 4.0 International. 


\title{
Poésies numériques tactiles : toucher les signes par la «main de l'œil », manipuler la matière à l'écran
}

\author{
Digital Tactile Poetry: To Touch Signs through the "Eye's Hand", to Manipulate \\ the Substance on the Touch Screen
}

Emmanuelle Pelard

1 Les néologismes " Poésure et Peintrie », résultant de la contraction agencée des termes poésie et peinture, furent repris, à la suite de Kurt Schwitters, à l'occasion d'une exposition présentée au Centre de la Vieille Charité par la Réunion des musées nationaux et les musées de Marseille du 12 février au 23 mai 1993, qui donnait à voir un certain nombre de productions graphiques et picturales où la poésie s'était faite image pour devenir un véritable fait plastique. La créativité terminologique pour nommer les pratiques et les œuvres hybrides, interartiales, conjuguant littérature et arts plastiques, n'a cessé de se développer depuis la seconde moitié $\mathrm{du} \mathrm{xx}^{\mathrm{e}}$ siècle en raison de la prolifération d'expérimentations intermédiales. Les expressions ou termes suivants en attestent: « sculptures-mots ", « tableaux-mots » (Cobra 1948), « poème idéogrammatique » (AlbertBirot 1918), «écripeinture» (Alechinsky 2004), «typoésie» (Peignot 1993), «photolittérature » (Edwards 2008), « poésie graphique» (Pelard 2012a), « ready-made poésie» (Théval 2015), et, plus récemment, s'agissant de la poésie numérique, « calligramme animé » (Bootz 2006, Saemmer 2011, Di Rosario 2012), « haiku interactif », «sonnet hypermédiatique» (Pelard 2016), etc. La nécessité de caractérisation de formes littéraires renouvelées, voire inédites, dont résulte cette inventivité lexicale manifeste le tournant essentiel qui l'a précédée, celui que constitue le penchant iconique, visuel et plastique de la littérature dans l'histoire littéraire.

2 Du Coup de dés jamais n'abolira le hasard de Mallarmé en 1897 aux poèmes numériques conçus pour être expérimentés sur des écrans tactiles, le potentiel visuel, voire 
synesthésique, de l'écriture a fait l'objet d'une multitude de propositions formelles. En effet, l'expérimentation plastique et visuelle du signe initiée par Mallarmé et développée par les avant-gardes poétiques du $\mathrm{xx}^{\mathrm{e}}$ siècle - notamment le lettrisme, le spatialisme, la poésie concrète, etc. - a été reprise et prolongée par la poésie numérique, à travers l'hypermédia ${ }^{1}$. L'image animée qui constitue souvent le texte numérique, parfois aussi corrélée à des phénomènes sonores, est aujourd'hui enrichie d'une dimension tactile. La littérature sort du livre, le texte se déverbalise, l'œuvre littéraire se meut en un processus expérientiel lié à une performance éphémère et à l'impossible reproductibilité du même, voire à une infinie variation de ses avatars. Si la dynamique expérimentale de la littérature épouse incontestablement un régime de l'image et s'inscrit dans un " pictorial turn", pour reprendre l'expression consacrée de W.J.T. Mitchell (1995), il semble toutefois que les débuts du XxI ${ }^{\mathrm{e}}$ siècle s'accompagnent aussi d'un tournant tactile grâce à l'interaction entre la main et le poème dans les œuvres créées pour être manipulées sur les écrans tactiles. Certaines pratiques poétiques expérimentales contemporaines testent les ressources du tactile dans sa capacité à faire ceuvre et explorent la possibilité pour le toucher de participer à l'élaboration du langage poétique et d'interroger les processus par lesquels se constitue la signification. Depuis 2010, des œuvres poétiques spécialement conçues pour écrans tactiles (smartphones, tablettes) mobilisent une manipulation des lettres, des mots et des signes afin que le poème advienne.

3 La coprésence du visuel et du tactile dans la formation du texte poétique ne s'organise pas selon une modalité concurrente, mais bien plutôt en concours, procédant d'une coimplication (Vouilloux 2013). Autrement dit, le tactile ne supplante pas l'image, mais opère comme relais de cette dernière et en prolongement du champ spécifiquement visuel. Dans cette perspective, il importe de considérer la fonction « haptique » de l'œil (Deleuze [1972] 1981 : 146), ce que Paul Valéry évoque poétiquement par l'expression « la main de l'œil » (1974 : II, 1301). Aussi, dans le cas des dispositifs tactiles d'une littérature écranique, la perception haptique prépare l'implication directe de la main, les opérations de manipulation des lettres à l'écran faisant partie de la production du texte. Si la relation entre l'œil et la main n'est pas nouvelle dans les pratiques artistiques plastiques et s'inscrit dans une certaine tradition de pensée - citons notamment les analyses de la sculpture (Herder 1770-1778), du bas-relief égyptien (Riegl 1901) ou de la peinture de Francis Bacon (Deleuze 1981) -, elle est en revanche peu explorée à l'endroit de la littérature. Pourtant, ce rapport visuel-tactile est désormais davantage mis en œuvre dans les pratiques expérimentales contemporaines de poésie (numérique, performance, spectacle vivant). Il s'agit ainsi dans cet article d'interroger la relation entre le « devenir image » et ce que nous qualifions de devenir tactile de la poésie numérique, à partir d'un corpus qui relève de différentes aires géographiques et linguistiques (États-Unis, France, Canada, Autriche, Mexique, Israël), et de se demander en quoi l'émergence de nouvelles formes poétiques s'appuie sur le paradigme de l'« image tactile» (Bergson 1932). Une approche sémiotique sera privilégiée afin d'envisager la mise en œuvre d'un dispositif poétique numérique haptique-tactile qui s'appuie notamment sur une interaction du corps, de l'écran et de l'écriture à l'origine de la sémiosis, sur le dialogue haptique-tactile et sur des processus rythmiques synesthésiques dans la poésie numérique tactile. 


\section{Le corps, l'écran et l'écriture : des « alliés substantiels² » dans les processus signifiants de la poésie numérique tactile}

Les pratiques de littérature numérique, de par leur nature même, celle d'être médiée par une interface, un logiciel, un ordinateur ou un écran, impliquent de considérer, de penser et d'interroger la relation entre le corps et l'écran - ce qui est encore plus pertinent à l'endroit des œuvres littéraires hypermédiatiques développées spécifiquement pour les écrans tactiles et dispositifs mobiles. Ces œuvres numériques à vocation tactile exploitent le potentiel technique et les fonctions spécifiques de l'écran tactile pour renouveler l'expérience littéraire et mettre encore davantage en relief la multiplicité sensorielle des textualités numériques que dans les œuvres conçues pour une lecture sur ordinateur et sur Internet. Les choix effectués en relation avec les caractéristiques et perspectives techniques du média et du support écranique participent à élaborer l'esthétique de l'œuvre. C'est pourquoi Paul A. Fishwick ouvre la voie à l'étude et à la conceptualisation d'une esthétique numérique dans Aesthetic Computing (2006). Plusieurs entreprises théoriques ont donné suite aux travaux de Fishwick, afin de déterminer quelles seraient les spécificités des poétiques et esthétiques numériques (voir Gervais et Saemmer 2011, Guilet et Pelard 2016) - à savoir, entre autres, la labilité, la fluidité du texte, le caractère éphémère de l'œuvre, les «figures de manipulation» (Bouchardon 2011: 45) qui supplantent les figures de rhétorique, une nouvelle rhétorique qui s'appuie sur un régime polysémiotique, l'interactivité et l'hypermédiaticité, etc.

Le texte n'existe plus seul ; il côtoie des images et il est intégré à des dispositifs qui l'animent, l'effacent ou l'opacifient à souhait. Ce sont des dispositifs qui en modifient substantiellement la forme ainsi que la texture, et qui exigent en fait de créer un nouveau vocabulaire, voire une nouvelle grammaire. (Gervais 2004 : 50)

La réflexion esthétique sur la littérature numérique implique donc de prendre en considération la variation des différents supports, formats et technologies. En l'occurrence, les écrans tactiles s'inscrivent dans la catégorie des "écrans reliés » (Gervais 2004 : 49), c'est-à-dire « un écran modifiable en temps réel, qui s'appuie sur des technologies d'inscription numériques et permet l'accès aux différents réseaux " (Archibald 2008: 160). Mais, plus précisément, l'écran relié dans le cas d'œuvres numériques conçues sous forme d'applications implique une «forme de re-stabilisation du texte, quand bien même interfacé par l'écran relié » (Guilet et Pelard 2016). « Avec les supports numériques mobiles, la qualité stable ou fluctuante du médium n'est pas un état subi du fait de la nature du médium, il résulte d'un choix poétique » (Guilet, Pelard 2016). En ce sens, il est manifeste que le dispositif technique (format de l'application, système d'exploitation) et les caractéristiques de sa configuration déterminent aussi l'esthétique de l'œuvre qui en résulte. C'est pourquoi la notion de dispositif est à envisager ici selon une double acception, c'est-à-dire le dispositif technique, la configuration du support tactile numérique, et le dispositif d'ordre esthétique qui organise l'œuvre littéraire numérique en format applicatif. Cette précaution semble d'autant plus pertinente s'agissant des «applications poétiques »- «nous recourrons à cette terminologie pour désigner les applications [pour tablettes tactiles et smartphones] qui prennent pour objet/ sujet le poème » (Pelard 2016). Cette approche double de la notion de dispositif s'articule différemment selon les pratiques numériques de la poésie, c'est-à-dire que le dispositif ne 
recouvre pas nécessairement la même réalité et n'opère pas de la même manière selon la nature du dialogue entre la dimension visuelle et la dimension tactile.

Dans la mesure où le dispositif a été très abondamment théorisé et commenté (Foucault, Lévi-Strauss, Deleuze, Lyotard, Aumont, Baudry, Agamben, et plus récemment Vouilloux, Ortel, etc.), nous ne reprendrons pas ici l'ensemble de son histoire conceptuelle. C'est l'acception suivante qui sera privilégiée dans notre analyse et définition du dispositif :

[...] un dispositif est un agencement résolument hétérogène d'énoncés et de visibilités qui lui-même résulte de l'investissement d'un ensemble de moyens appelé à fonctionner stratégiquement au sein d'une situation (d'un champ de forces) donnée. [...] Les dispositifs opèrent à tous les niveaux sémiotiques des textes et des images [...]. La partition entre énoncés et visibilités ne se superpose pas à celle entre les textes et les images [...] Enfin, ce qu'on appelle « œuvre » agence ou monte plusieurs dispositifs : l'œuvre elle-même fait dispositif. (Vouilloux 2008: 28-31)

7 En outre, afin de saisir le fonctionnement du dispositif des applications poétiques, il est nécessaire de prendre en compte «la technologisation du plan d'expression» (Zinna 2004 : 292) à l'œuvre dans les textualités numériques. Effectivement, « [1]a notion d'objetécriture ouvre les frontières du texte par l'intégration des fonctions de l'objet d'usage. Peut-être cette ouverture n'aurait-elle pas été nécessaire si la technologisation du plan d'expression n'avait pas soudé définitivement l'univers du discours et celui des objets » (Zinna 2004 : 292). Dès lors que les écritures numériques relèvent d'une logique d'objet et que l'approche linguistique de l'écriture et celle de l'évolution technologique sont indissociables, le support matériel devient de plus en plus un lieu symbolique (Zinna 2004: 292). C'est pourquoi s'agissant de la poésie numérique tactile, il importe d'envisager le fonctionnement du dispositif technique dans sa relation avec le dispositif esthétique, notamment eu égard à la dimension haptique ou tactile, c'est-à-dire à l'engagement du corps à travers l'expérience de perception / réception de l'ordre visuel et tactile. Recouvrant tant des applications poétiques (poème en format application) que de la poésie action numérique sous forme de performance scénique, la poésie numérique tactile resitue l'attention sur le corps soit par l'implication directe de la main (manipulation tactile sur l'écran), soit par la démonstration de la performance du corps et par le jeu sur la perception haptique.

C'est particulièrement manifeste dans un exemple de poésie-performance actuel qui se fonde sur une utilisation du numérique mettant en relief le corps dans sa relation avec les mots et les signes graphiques, par une projection des signes sur le corps en mouvement sur la scène ou par une mise en scène du corps dans l'espace comme moyen de créer du sens. Il s'agit de la poésie action numérique de HP Process ${ }^{3}$, un duo de poètes-performeurs numériques français (Hortense Gauthier et Philippe Boisnard), qui se présente sous une kyrielle de désignations génériques et formelles telles que poésie action, performance numérique, pure-data, poésie visuelle, poésie sonore, vidéo-poésie, installation digitale, dispositif interactif et e-poetry ou poésie numérique. Hp Process expérimente le potentiel du corps comme surface signifiante, explore la matière des signes, les relations entre langage, espace-temps et corporéité, mais aussi les possibilités rythmiques de l'algorithme, les dispositifs d'écriture et les relations en réseaux, à la croisée de la quête poétique, de la performance scénique et du spectacle hypermédiatique. Si chaque performance est unique, il n'en demeure pas moins qu'un certain nombre de caractéristiques esthétiques sont réitérées, à savoir une représentation fondée sur une expérience synesthésique, une interrogation de la construction du sens à travers une 
mobilisation et une saturation des signes générées de manière algorithmique et projetées sur différentes surfaces, et corrélativement la métaphore des écrans envisagés en tant que supports de l'écriture (le corps œuvrant aussi comme écran réceptacle de l'écriture), enfin la symbolisation du corps. Non seulement le corps agit comme surface qui reçoit et inscrit les signes graphiques qui défilent incessamment, mais il est aussi en lui-même signifiant. En ce sens, nous rejoindrons la perspective sémiotique de Jacques Fontanille dans Corps et sens (2011) sur le rôle du corps dans les processus signifiants, à travers son implication dans la production du discours, dans les manifestations sensibles et perceptives, comme base de la sémiosis. «En effet, par l'intermédiaire de la sensation et de la perception, le corps, placé au cœur de la production du sens, est susceptible de nous fournir des modèles de la schématisation, de la transformation et de la mise en séquence des figures " (Fontanille 2011 : 53-54). Si le corps est essentiel dans la constitution du sens, les pratiques poétiques qui présentent le corps comme moyen et lieu signifiants appellent à faire une corrélation entre le corps comme médium de la performance poétique et la production du sens qui en découle. Ainsi, partant du postulat que les interactions entre les corps - le « corps-actant » et le « corps-sensible » (Fontanille 2011: 155) - sont au cœur de l'élaboration de la signification, on peut envisager la démarche de HP Process comme étant une investigation artistique de cette dimension signifiante du corps. En effet, dans le cadre des performances de HP Process, le corps des performeurs se meut à travers l'espace scénique, traversant et recevant les projections de signes générées numériquement; non seulement le corps agit, "corps-actant ", dans l'acte chorégraphique, se situant ainsi au cœur du dispositif esthétique, mais il réinvestit d'une signification l'action de dislocation du langage et de désémantisation opérée par la projection de signes d'écriture de manière aléatoire. Qui plus est, les performeurs sont très dénudés, parfois nus, ce qui accentue la perception du corps comme une surface directe d'inscription de l'écriture, sans autre médiation, lui conférant la valeur d'un écran (support habituel des écritures et images numériques) : un écran de chair. La mise en scène du corps, l'appréhension du corps comme écran de l'image graphique numérique animée, projetée sur la chair réfléchissante de la performeuse, place au centre de la performance la dimension haptique.

\section{La relation du couple haptique-tactile : substrat du dispositif poétique numérique}

Certaines pratiques telles que La Poésie à 2-mi mots (ALIS, Bouchardon 2012) font se croiser la performance poétique numérique sur scène et l'utilisation par le public d'une application poétique sur écran tactile, fondée sur le même procédé poétique que celui mis en œuvre par les performeurs. Ces œuvres explorent la dimension visuelle du texte poétique en investissant les possibilités techniques et esthétiques de l'image animée, plus spécifiquement de l'image numérique, tout en approfondissant la dimension haptique jusqu'à embrasser complètement le champ du tactile. En effet, dans La Poésie à 2-mi mots le geste du lecteur-utilisateur pour séparer le mot qui apparaît à l'écran permet d'en former un autre, et c'est de leur mise en relation grâce à la manipulation tactile que naît la signification du poème. Le dispositif poétique ${ }^{4}$, en ce sens, doit être dès lors pensé en relation avec la dimension haptique-tactile, puisque c'est le point de convergence de la démarche esthétique et de la configuration technique du support caractérisant en grande partie la poésie numérique. 
10 Il s'agit, ainsi que le propose Henri Maldiney relatant l'analyse d'Aloïs Riegl, de considérer « deux possibilités du regard, deux structures significativement différentes de l'avoir en vue, deux types de vision articulés à deux types d'espace artistique, qu'il nomme respectivement optique et haptique " (Maldiney 1973: 194). Dans la poésie numérique tactile, la sensation éprouvée par le lecteur-spectateur-utilisateur naît de la rencontre avec une surface, d'une implication du toucher, que la relation soit directe et intentionnelle (tactile) ou indirecte et éventuellement circonstancielle (haptique). La vision haptique est une perception de la proximité, de même que le toucher se définit par une appréhension du proche. «Dans la zone spatiale des proches, sur laquelle nous sommes en prise directe, le regard procédant comme le toucher éprouve au même lieu la présence de la forme et du fond (Maldiney 1973 : 195). La vision haptique permet de percevoir et d'apprécier le relief, le volume et la matière des signes et des formes du poème numérique, ce qui s'avère essentiel dans la mesure où ce type de pratique poétique interroge, met en scène et bouleverse la matérialité du langage afin de manifester la place $\mathrm{du}$ corps dans les processus signifiants. L'impératif théorique qui se pose est de déterminer comment la composante haptique/tactile s'inscrit dans le dispositif poétique. Dans cette perspective analysons la Poésie à 2-mi mots, technique poétique inventée par Pierre Fourny en 2000, qui consiste à découper un mot imprimé sur un papier d'un trait horizontal et à assembler chaque moitié du mot à d'autres moitiés de mots, constituant à leur tour des mots. Le poème s'élabore dans cette recomposition typographique des mots et dans leur association les uns avec les autres. Il s'agit d'une poésie visuelle, d'une poésie de la typographie. «Le mot écrit doit devenir objet concret, ou être associé à un objet concret, et être manipulé physiquement, ou virtuellement, pour exprimer un sens qui n'apparaîtrait pas sans ce déportement: en bref : la Poésie à 2-mi mots produit des spectacles ${ }^{5}$. Cette pratique poétique a ensuite été mise en scène dans plusieurs spectacles montés par Pierre Fourny ${ }^{6}$. Ce dernier précise qu'il envisage la Poésie à 2-mi mots non pas uniquement en tant qu'une entreprise littéraire, mais aussi comme la " conséquence d'un travail scénique ${ }^{7}$ ", ce qui lui confère une mixité générique. D'une pratique utilisant le papier, la Poésie à 2-mi mots a été remédiée et se fait maintenant sur ordinateur. Elle devient dès lors une poésie générative produite par un logiciel, une typographie animée. Elle renouvelle également la pratique scénique, puisque Fourny utilise l'ordinateur sur scène. À travers ce processus de remédiation et une collaboration, co-création, entre Fourny, l'ALIS, Serge Bouchardon, l'Université de Technologie de Compiègne et le collectif artistique i-Trace (dans le cadre d'un projet en recherchecréation), la Poésie à 2-mi mots devient Typomatic, la Séparation ${ }^{8}$ et Typoring, des applications de poésie à 2-mi mots pour écrans tactiles mobiles. La Séparation et le Typomatic font aussi partie du spectacle de Poésie à 2-mi mots dans la mesure où les performeurs sur scène, Pierre Fourny et Serge Bouchardon, tout en manipulant physiquement les mots réinventés sur des cartons papier, puis virtuellement en produisant d'autres mots par écran et ordinateur interposés, incitent le public à utiliser l'application du Typomatic pour produire lui-même son poème à 2-mi mots. Les spectateurs peuvent imprimer ce qu'ils ont produit à la fin du spectacle. L'investigation de la physicalité du poème et des signes graphiques repose ici sur une conjugaison des différentes modalités du prendre corps et de l'articulation du visuel et du tactile, sans évoquer la pluralité des formes de la spectacularisation et de la scénographie.

La scène et les écrans sont des espaces «parents » dès lors qu'on les envisage dans

la perspective de manipulation d'objets, de signes linguistiques et d'images, de sons, comme le pratique ALIS. [...] Dans le cas des écritures numériques, la fonction 
scénique de l'écran est renforcée par le fait que les actions programmées le sont pour l'espace que définit l'écran [...]. C'est aussi parce que le sens est en train de se produire, dans l'écran, que cet écran fait scène, lieu du spectacle. [...] Et les petits écrans numériques, interactifs et légers, le font encore de manière plus flagrante car ils appellent, sur scène, le geste et facilitent/encouragent leur propre manipulation. [...] Dans le cadre des performances de poésie numérique données, il s'agit d'articuler les manipulations dans l'espace de l'écran (l'écran comme scène) et les manipulations des écrans eux-mêmes dans l'espace scénique (l'écran sur scène), mais aussi de penser la présence du spectateur avec son désir d'interaction et avec son propre écran-scène dans la poche. (Bouchardon 2016)

11 Cette pratique numérique de la poésie repose sur des stratégies de manipulation qui sont d'autant plus fortes, ou à tout le moins évidentes, qu'elles impliquent directement le toucher, la préhension tactile des mots et des signes graphiques par la main ${ }^{9}$. Aussi à travers une expérience tactile du poème et une représentation haptique de l'écriture qui met en lumière la matérialité du signe, le corps (du performeur, du lecteur-utilisateur) et l'écran deviennent des surfaces signifiantes. Enfin, les effets poétiques et la signification du poème se forment grâce à la suggestion haptique et/ou la manipulation tactile, à l'origine (en partie) de la formation du rythme.

\section{Des processus rythmiques synesthésiques dans la poésie tactile numérique : voir et toucher la matière en mouvement}

Un autre des éléments essentiels qui caractérisent les œuvres poétiques hypermédiatiques tactiles est l'expérience esthésique, due à la multiplicité et à la combinaison des différentes dimensions sensorielles de l'œuvre. En effet, il y a une interaction entre le son, l'image animée, les effets haptiques et la manipulation tactile qui concourent à produire la signification de l'œuvre. Cette composition sensorielle du poème, voire sensori-motrice lorsque les opérations de manipulation de la tablette tactile sont particulièrement importantes, procède d'une dynamique d'organisation qui n'est autre que le rythme. Le cycle d'applications poétiques de Piringer dont les titres sont souvent évocateurs de l'objet du poème-Konsonant, Unicode, Tiny Poems, Unimator, RealBeat, Gravity Clock ${ }^{10}$ - propose des expériences sonores et visuelles ${ }^{11}$ des signes alphabétiques et typographiques (comme dans les pratiques de la poésie concrète au xx siècle), mais aussi une expérimentation tactile de manipulation des signes à même l'écran, afin de renouveler la manière de faire œuvre poétique et d'engendrer la signification. Les «figures de manipulation » envisagées dans une perspective sémiorhétorique (Bouchardon 2011 : 45) constituent l'une des caractéristiques essentielles de la littérature numérique. En outre, dans le cadre des œuvres hypermédiatiques conçues pour écrans tactiles, l'impact des «figures de manipulation» dans les processus signifiants est d'autant plus grand que l'interaction avec le support est directe, et la main, le contact, l'action tactile se font les opérateurs et catalyseurs essentiels de la signification poétique. Ainsi, dans l'application abcdefghijklmnopqrstuvwxyz, mettant en scène une génération de lettres qui traversent l'écran de manière aléatoire, le lecteur peut, en manipulant le signe, modifier le rythme et la trajectoire de ce dernier sur le plan graphique et visuel ainsi que l'émission de son qui l'accompagne. Les traces des différentes manipulations qui forment le parcours des lettres s'inscrivent ensuite graphiquement et demeurent à l'écran. L'intervention tactile du lecteur-utilisateur, dans 
la mesure où elle modifie la configuration poétique, produit corrélativement des effets esthétiques, crée une image graphique des processus rythmiques et fait advenir la signification de la performance poétique de l'application. Plusieurs types de geste peuvent être à l'origine des effets poétiques de nature rythmique : secouer l'écran pour engendrer un texte, manipuler les signes sur l'écran, interrompre le flux graphique, le rythme, les phrases par le contact des doigts, placer l'écran au-dessus de sa tête pour voir d'en bas une pluie de mots qui tombe, animée à l'écran, etc. Le poète numérique choisit parmi un panel de types de fonction et de gestes possibles selon le format pour créer la structure poétique.

L'écran tactile multi-touch possède l'avantage d'autoriser des opérations plus directes qu'un dispositif de pointage comme le stylet ou la souris. Il implique une pluralité d'IHM basées sur un panel inédit de gestes que les logiciels iOS ou Android interprètent et régissent. [...] C'est le système d'exploitation qui définit donc les différents gestes exécutables, puis les développeurs d'applications sélectionnent au sein de ce panel les gestes nécessaires à l'utilisation de leurs créations. (Guilet 2016)

13 Les possibilités du dispositif technique (l'écran tactile, les systèmes d'exploitation Android et iOS et les technologies qu'ils impliquent et offrent) configurent et préfigurent le dispositif esthétique, poétique, qui lui-même repose ensuite sur une manipulation, une mise en œuvre et une expérimentation par le lecteur-utilisateur.

dialogue entre les possibilités techniques et les choix esthétiques crée les conditions pour une expérience synesthésique du rythme, qui participe à l'élaboration de la signification poétique. C'est le cas du cycle de Poetry for Excitable Mobile Media de Jason Edward Lewis (États-Unis et Canada, 2010-2015), de celui de Concrete poetry for mobile device de Jörg Piringer (Autriche, 2010-2016) ainsi que de l'application He liked thick word soup appartenant au cycle Chronotext d'Ariel Malka (Israël, 2010-2015). Ces œuvres s'apparentent toutes à une version hypermédiatique de la poésie concrète en raison de leurs propositions polysémiotiques et multisensorielles de la graphie (alphabétique, hébraïque, idéogrammatique, etc.). Les différents principes d'animation, de génération et de manipulation des signes représentent les effets rythmiques essentiels dans ces œuvres. Ainsi, dans Konsonant ${ }^{12}$, Piringer permet au lecteur-utilisateur de dessiner à même l'écran une ligne qui dès lors confère le rythme et la trajectoire aux lettres de l'alphabet générées automatiquement, mais de manière aléatoire par l'algorithme. Aussi, le lecteur-utilisateur peut interrompre l'organisation du flux de lettres à tout moment et le réorganiser, ce qui modifie le sens de l'œuvre en perpétuelle constitution. Une logique rythmique similaire est à l'œuvre dans les P.o.E.M.M.s de Jason Edward Lewis, notamment dans Speak ${ }^{13}$ ou encore dans No Choice About the Terminology ${ }^{14}$, où un défilé continu de mots et de constituants de phrases peut être défait et refait par le lecteur, les combinaisons de segments de phrases en mouvement étant dès lors infiniment variées et variables. L'altération du rythme par génération configure les processus signifiants à l'origine du poème.

Un procédé relativement semblable organise l'application He liked thick word soup ${ }^{15} \mathrm{~d}$ 'Ariel Malka, qui propose une génération aléatoire de fragments d'Ulysse de James Joyce. Les phrases du roman défilent à l'écran et lorsque le lecteur-utilisateur touche un mot ou une lettre, le rythme est modifié et le point de contact à l'écran avale la phrase dans une lettre pour qu'une autre apparaisse. La cristallisation de la phrase sur une lettre modifie complètement la signification et la diégèse de l'œuvre de Joyce, faisant des signes qui composent son écriture des objets graphiques, visuels, haptiques, tactiles. Toute l'originalité de cette œuvre réside dans une mise en hypermédia du principe 
d'intertextualité à travers la reprise du texte d'Ulysse, le déploiement d'une intertextualité hypermédiatique où le rythme joue un rôle sémiotique et poétique important. Les précédentes réalisations numériques de Malka, réunies dans le projet Chronotext ${ }^{16}$, touchaient à la plasticité des signes d'écriture hébraïques grâce à une mise en relief en 3D et une animation numérique de textes israéliens où il s'agissait d'explorer la matière de la graphie hébraïque. L'expérimentation du signe et du rythme de l'écriture, qu'il s'agisse de l'hébreu ou de l'anglais, est primordiale. Cette tentative d'écriture en 3D est aussi ce que Piringer développe dans son application poétique Tractacus infinitus, où les mots et les signes générés aléatoirement modifient leur signifiant graphique (déformation du tracé de la lettre, surenchère de matière qui envahit l'écran, faisant ainsi $\mathrm{du}$ signe graphique un signe iconique ou plastique $^{17}$, voire transformation de la typographie en ligne plastique abstraite, etc.). C'est le contact du doigt du lecteurutilisateur qui, à travers la modification du rythme, engendre toutes ces transformations sémiotiques, permet la réalisation actualisée de l'œuvre et participe à créer la signification poétique. La dimension tactile, et pas seulement celles de l'ordre sonore et visuel, participe aussi du processus rythmique à l'origine du poème. Afin de penser cette articulation entre une dimension plastique-graphique du rythme plus accentuée que dans des formes de poésie traditionnelles, la dimension sonore et une approche haptique, voire complètement tactile, il est nécessaire d'envisager une nouvelle approche du rythme. Si les poésies visuelle et graphique avaient déjà posé cette nécessité du renouvellement théorique de la notion de rythme afin de pouvoir saisir la particularité de leur esthétique - ce pourquoi nous avons proposé une théorie du « rythme graphique ${ }^{18} »$ (Pelard 2012b : 176) -, il n'en demeure pas moins que cette voie d'investigation doit être poursuivie et approfondie à l'endroit de la poésie numérique, en raison de la complexité des processus synesthésiques d'organisation de la matière qui la caractérisent. Un manque théorique concernant cet élément essentiel de la poétique des poésies numériques nous a amené à compléter la notion de rythme graphique. Il s'agit d'« une dynamique d'organisation graphique non régulière indissociable du déploiement de la signification, fondée sur des retours minimaux de points qualifiés dans la configuration graphique et qui s'appuie sur une dynamique spatiale et temporelle de rétention et de protention sur le plan graphique » (Pelard 2012b: 176). Les œuvres hypermédiatiques poétiques qui reposent sur un processus de génération algorithmique et sur une expérimentation des signes et $\mathrm{du}$ langage procèdent de cette dynamique d'organisation du mouvement. Plus spécifiquement concernant la poésie numérique tactile, le rythme de nature graphique est aussi lié à l'implication de la dimension tactile, c'est-à-dire de l'interférence entre le flux de la génération et l'intervention du doigt et de la main du lecteur-utilisateur sur ce dernier et ce qu'il en résulte.

Dans les deux cas, que le rythme soit généré par le logiciel ou modifié par l'utilisateur, la dynamique d'organisation graphique est non régulière et engendre une rétention et une protention grâce à la présence de points qualifiés, avec des retours minimaux de points qualifiés (la lettre -a est un point qualifié et elle est répétée) et d'intervalles (un autre signe alphabétique est reproduit et contraste avec une lettre différente). C'est cela qui crée des processus de comparaison et différenciation dans les séquences graphiques qui se forment au fur et à mesure à l'écran. La dynamique d'organisation non régulière du mouvement des lettres est liée au déploiement de la signification du poème : à savoir la réflexion sur le signe, l'expérimentation des capacités de l'alphabet à performer autrement le son et l'image, à produire un sens sans passer par l'articulation linguistique et syntaxique grâce à laquelle le langage naturel produit habituellement le sens. (Pelard 2016) 
Aussi, des signes plastiques et iconiques peuvent constituer des points qualifiés opérant des liaisons et des intervalles. Les liaisons peuvent ne pas procéder d'une succession spatiale immédiate, mais du retour de certaines formes plastiques, créant alors un mouvement rétentionnel. Une dynamique protentionnelle émerge des segmentations et des ruptures dans la morphologie et dans la structure de la configuration graphique ou dans la forme des signes iconiques (Pelard 2012b : 177). La manipulation tactile du signe participe du rythme et l'expérience esthésique (à travers le concours des perceptions visuelle haptique, tactile et sonore) est au fondement du poème en devenir. La présence de processus synesthésiques sur lesquels reposent les effets et la signification poétiques caractérise les œuvres hypermédiatiques conçues pour écrans tactiles. En l'occurrence, l'application poétique Concretoons cartuchera $01^{19}$ de Benerva (Minerva Reynosa, Benjamin R. Moreno, Mexique, 2015) présente une poésie concrète numérique revue sous forme de jeu vidéo. L'esthétique graphique des jeux vidéo des années 1980 (Mario Bros, 1983, Nintendo) est réutilisée ici ainsi que la logique de progression du jeu : il faut franchir les niveaux pour pouvoir finir de composer le poème en devenir. Les différentes actions et commandes font apparaitre des signes graphiques et des signes plastiques ou iconiques qui eux-mêmes dévoilent le texte poétique, se formant au fur et à mesure de la partie. Le retour de certains signes iconiques ou de certaines lettres et leurs effets corrélatifs créent une dynamique de rétention, permise par des intervalles et des ruptures dans les figures qui apparaissent à l'écran sur le plan spatial; cette alternance entre mouvements protentionnel et rétentionnel organise le rythme qui par effets de liaison et déliaison produit le texte et la signification poétiques. Toucher et manipuler les signes de la matière en motion constitutive du poème représente un des aspects essentiels du poème numérique tactile. L'intervention directe du toucher, de la main, et pas seulement de la «main de l'œil» (Valéry 1974: II, 1301), autrement dit de la perception haptique, contribue à la formation des processus rythmiques synesthésiques du poème, qui sont partie prenante du dispositif poétique numérique haptique-tactile.

17 En définitive, les points de convergence qui se manifestent dans les cas étudiés, mais également dans un certain nombre d'œuvres qui s'inscrivent dans ce qu'on peut qualifier de poésie numérique tactile, permettent de formuler l'hypothèse d'un dispositif poétique numérique haptique-tactile. Ce dispositif se définit par une représentation haptique-tactile de l'écriture pour suggérer la matérialité du signe graphique, permise par la présence de "figures de manipulations» (Bouchardon 2011: 45) et l'implication de la main et du corps dans l'expérience poétique grâce aux possibilités du dispositif tactile (l'écran tactile mobile), mais il se caractérise aussi par des processus sémiosiques reposant sur la mobilisation du toucher dans le poème, par un lien direct entre la création de l'effet poétique, la production de la signification et la suggestion haptique et/ou le geste tactile ainsi que par une expérience esthésique et des processus rythmiques synesthésiques où le corps et l'écran constituent des surfaces signifiantes.

18 Le dispositif poétique numérique haptique-tactile résulte d'un geste esthétique fondé sur l' hypermédiaticité et sur une sensorialisation accentuée du texte. Les informations et impressions que cette sensation du toucher protéiforme - issue du dialogue de la vision haptique et du contact direct de la main avec l'écran - apporte au lecteur-utilisateur constituent l'essence de l'émotion esthétique. L'œuvre poétique se crée à travers 
l'expérience esthésique. Et c'est dans ce nœud de l'esthésie où se croisent le visuel et le tactile que le "devenir image» de la poésie se conjugue à ce que nous qualifions de devenir tactile - qui se définirait comme un passage de la poésie à un avatar tactile de la mise en scène textuelle, de la performance poétique, reposant sur des dispositifs écraniques tactiles, où le corps fait activement, directement, partie du dispositif poétique et des processus signifiants. Si l'émergence de formes poétiques inédites est manifeste dans ce tournant tactile de la poésie, il n'en demeure pas moins qu'un certain nombre d'applications poétiques revisitent des formes fixes de la poésie (sonnet, haïku) ou des types poétiques des avant-gardes du $\mathrm{Xx}^{\mathrm{e}}$ siècle (poésie concrète, poésie sonore). L'histoire esthétique de la poésie est donc re-vue, re-maniée par les poètes numériques contemporains à l'aune de la révolution épistémologique numérique et de ses innombrables possibles.

\section{BIBLIOGRAPHIE}

Albert-Birot, Pierre, [1918] 1980, « Les Éclats, poème idéogrammatique », SIC, Paris, Jean-Michel Place.

Alechinsky, Pierre, 2004, Des deux mains : traits et portraits, Paris, Mercure de France.

Archibald, Samuel, 2008, Le Texte et la Technique. La lecture à l'heure des nouveaux médias, Thèse de doctorat, Université du Québec à Montréal.

Bergson, Henri, [1982] 2001, Les Deux Sources de la morale et de la religion (1932), Euvres complètes, Paris, PUF.

Bootz, Philippe, 2006, « Vers de nouvelles formes en poésie numérique programmée ? », RiLUnE, $\mathrm{n}$ ० 5, p. 19-35.

Bouchardon, Serge, 2011, « Des figures de manipulation dans la création numérique », Protée, vol. $39, \mathrm{n}^{\circ} 1$, p. 37-46.

Bouchardon, Serge, 2016, « Les écrans mobiles et tactiles : des lieux de spectacle vivant ? Le cas de la Poésie à 2-mi mots ", Poétiques et esthétiques numériques tactiles : Littérature et Arts, Cahiers virtuels du Laboratoire NT2, $\mathrm{n}^{\circ}$ 8, [En ligne], http://nt2.uqam.ca/fr/cahiers-virtuels/article/lesecrans-mobiles-et-tactiles-des-lieux-de-spectacle-vivant-le-cas-de-la, consulté le 8 mai 2018.

Collectif, 1993, Poésure et peintrie : d'un art, l'autre, Marseille, Paris, Réunion des musées nationaux.

Deleuze, Gilles, [1972] 1981, Logique de la sensation, Paris, Éditions de la Différence.

Di Rosario, Giovanna, 2012, « Electronic Poetry: How to Approach It? », Texto !, vol. 17, $\mathrm{n}^{\text {os }} 1-2$, n. p.

Draguet, Michel (dir.), 1994, Cobra en Fange, Vandercam-Dotremont : Dessin-Écriture-Matière ( 1958-1960), Bruxelles, Éditions Snoeck, Université Libre de Bruxelles.

Edwards, Paul, 2008, Soleil noir. Photographie et littérature des origines au surréalisme, Rennes, PUR.

Fishwick, Paul, 2006, Aesthetic Computing, Cambridge, MA, MIT Press. 
Fontanille, Jacques, 2011, Corps et sens, Paris, PUF.

Gervais, Bertrand, 2004, « Naviguer entre le texte et l'écran. Penser la lecture à l'ère de l'hypertextualité ", Les défis de l'édition à l'ère de l'hypertexte, Lyon, Enssib, p. 49-68.

Gervais, Bertrand et Saemmer, Alexandra (dir.), 2011, Protée, vol. 39, n 1, « Esthétiques numériques », [En ligne], https://www.erudit.org/fr/revues/pr/2011-v39-n1-pr5004899/, consulté le 8 mai 2018.

Guilet, Anaïs, 2016, « Préambule historique et technique sur les supports tactiles et deux de leurs systèmes d'exploitation (iOS et Android) », Poétiques et esthétiques numériques tactiles : Littérature et Arts, Cahiers virtuels du Laboratoire NT2, $\mathrm{n}^{\circ}$ 8, [En ligne], http://nt2.uqam.ca/fr/cahiers-virtuels/ article/preambule-historique-et-technique-sur-les-supports-tactiles-et-deux-de, consulté le 8 mai 2018.

Guilet, Anaïs et Pelard, Emmanuelle (dir.), 2016, Poétiques et esthétiques numériques tactiles : Littérature et Arts, Cahiers virtuels du Laboratoire NT2, $\mathrm{n}^{\circ}$ 8, [En ligne], http://nt2.uqam.ca/fr/ cahiers-virtuels/poetiques-et-esthetiques-numeriques-tactiles-litterature-et-arts, consulté le 8 mai 2018

Guilet, Anaïs et Pelard, Emmanuelle, 2016, « Esthétiques et poétiques des œuvres hypermédiatiques pour écrans tactiles : introduction », Poétiques et esthétiques numériques tactiles : Littérature et Arts, Cahiers virtuels du Laboratoire NT2, $\mathrm{n}^{\circ}$ 8, [En ligne], http://nt2.uqam.ca/fr/ cahiers-virtuels/article/esthetiques-et-poetiques-des-oeuvres-hypermediatiques-pour-ecranstactiles, consulté le 8 mai 2018.

Hanna, Christophe, 2010, Nos dispositifs poétiques, Paris, Questions Théoriques.

Herder, Johann Gottfried, [1778] 2002, Sculpture: Some Observations on Shape and Form, in Pygmalion's Creative Dream, trad. Laura Veit, Chicago, University of Chicago Press.

Maldiney, Henri, 1973, Regard, parole, espace, Lausanne, Éditions L'Âge d'Homme.

Mitchell, W.J.T., 1995, Picture Theory, Chicago, University of Chicago Press.

Peignot, Jérôme, 1993, Typoésie, Paris, Imprimerie nationale éditions.

Pelard, Emmanuelle, 2012a, « Fantasmes graphiques au péril du sens : le logogramme de Christian Dotremont, l'idéogramme imaginaire d'Henri Michaux ", Elseneur, Revue annuelle de littérature, $\mathrm{n}^{\circ}$ 27, p. 113-120.

Pelard, Emmanuelle, 2012b, La Poésie graphique : Christian Dotremont, Roland Giguère, Henri Michaux et Jérôme Peignot, thèse de doctorat, Université de Montréal, Université Paris-Sorbonne.

Pelard, Emmanuelle, 2016, « Poétique de la poésie numérique pour écrans tactiles ", Poétiques et esthétiques numériques tactiles : Littérature et Arts, Cahiers virtuels du Laboratoire NT2, $\mathrm{n}^{\circ} 8$, [En ligne], http://nt2.uqam.ca/fr/cahiers-virtuels/article/poetique-de-la-poesie-numerique-pour-ecranstactiles, consulté le 8 mai 2018.

Riegl, Aloïs, [1901] 2000, L'Industrie d'art de l'époque romaine tardive, Paris, Éditions Macula.

Saemmer, Alexandra, 2007, Matières textuelles sur support numérique, Saint-Étienne, Publications de l'Université de Saint-Étienne.

Théval, Gaëlle, 2015, Poésies ready-made, XX'e-XXI siècles, Paris, L'Harmattan.

Tore, Gian Maria, 2006, « Alessandro ZINNA, Le Interfacce degli oggetti di scrittura. Teoria del linguaggio e ipertesti, Roma, Meltemi, 2004, 311 pages ", Actes Sémiotiques, no 104-106, [En ligne], http://epublications.unilim.fr/revues/as/2155, consulté le 8 mai 2018. 
Valéry, Paul, 1974, Cahiers II, Paris, Gallimard, coll. « Pléiade ».

Vouilloux, Bernard, 2008, « Du dispositif », dans Philippe Ortel (dir.), Discours, image, dispositif. Penser la représentation II, Paris, L'Harmattan.

Vouilloux, Bernard, 2013, « Lire, voir. La co-implication du verbal et du visuel », Textimage, Revue d'étude du dialogue texte-image, Varia 3, [En ligne], http://www.revue-textimage.com/07_varia_3/ vouilloux1.html, consulté le 8 mai 2018.

Zinna, Alessandro, 2004, Le Interfacce degli oggetti di scrittura. Teoria del linguaggio e ipertesti, Rome, Meltemi.

\section{NOTES}

1. L'exploration du potentiel visuel de l'écriture au sein du poème se manifeste dès l'Antiquité avec les calligrammes de Simmias de Rhodes ( $V^{\mathrm{e}}$ siècle av. J.-C.) qui donnent lieu à une longue tradition de recherche de l'image à travers l'écriture poétique dans les littératures européennes. C'est ce dont témoignent particulièrement le moment mallarméen et le temps fort des avantgardes dadaïste et surréaliste. Les origines de la poésie numérique résident dans un approfondissement des logiques combinatoires des mots telles qu'envisagées par L'Oulipo ainsi que des expérimentations de la lettre et de la spatialité de l'écriture réalisées par la poésie visuelle au $\mathrm{xx}^{\mathrm{e}}$ siècle. La filiation évolue dans le passage d'une intermédialité caractéristique des formes précédemment mentionnées à l'hypermédialité de celles de la littérature numérique. Ce passage s'effectue dans le changement de support et de dispositif (du papier à l'écran), dans l'évolution d'une image fixe à une image animée, dans une production de l'écriture par l'algorithme et par des stratégies collaboratives impliquant le lecteur dans le processus de création / réception du texte grâce à l'hypertexte, au format applicatif, etc.

2. René Char, Feuillets d'Hypnos, Paris, Gallimard, 1946.

3. Voir le site de HP Process, http://databaz.org/hp-process/, consulté le 8 mai 2018.

4. Si les «dispositifs poétiques» tels que définis par Christophe Hanna (2010) permettent de saisir un ensemble d'aspects de la poésie expérimentale contemporaine, il reste que la composante numérique et la spécificité tactile des formes que nous évoquons doivent être davantage précisées, notamment quant à la relation entre le dispositif technique, les effets esthétiques et les processus sémiotiques.

5. Voir http://www.olats.org/livresetudes/basiques/artstechnosnumerique/7_basiquesATN.php, consulté le 8 mai 2018.

6. Quelques noms de spectacles montés et présentés par l'ALIS : La Coupure (2010), Le cirque de mots 2.0 (2013).

7. Voir le site de l'ALIS, https://www.alislab.fr/, consulté le 8 mai 2018.

8. Collaborateurs de La Séparation: Pierre Fourny et Hélène Caubel (ALIS), Serge Bouchardon, Guillaume Jacquemin (Buzzing Light), Alexis Schad, Luc dall'Armellina.

9. Ce qui est encore davantage le cas que pour les formes non tactiles de la poésie hypermédiatique.

10. Voir les navigations filmées des différentes applications poétiques sur le site de Jörg Piringer, http://apps.piringer.net/, consulté le 8 mai 2018.

11. À l'exception près qu'il s'agit là d'une image animée et interactive.

12. Voir les navigations filmées des différentes applications poétiques sur le site de Jörg Piringer, http://apps.piringer.net/konsonant.php, consulté le 8 mai 2018.

13. Voir les navigations filmées des différentes applications poétiques sur le site de Jason Edward Lewis, http://www.poemm.net/projects/speak.html, consulté le 8 mai 2018. 
14. Opcit, http://www.poemm.net/projects/choice.html, consulté le 8 mai 2018.

15. Voir sur le site Chronotext d'Ariel Malka, http://chronotext.com/WordSoup/, consulté le 8 mai 2018.

16. Voir sur le site Chronotext d'Ariel Malka, http://chronotext.org/scriptorium/, consulté le 8 mai 2018.

17. Selon la terminologie et les définitions données par le Groupe $\mu$ dans le Traité du signe visuel, Pour une rhétorique de l'image (1993).

18. La théorie du «rythme graphique» a été élaborée à partir du modèle développé par Lucie Bourassa pour le rythme linguistique, dans Rythme et sens, des processus rythmiques en poésie contemporaine (1993).

19. Concretoons cartuchera 01: Mammut - Minerva Reynosa, https://itunes.apple.com/es/app/ concretoons-cartuchera-01/id1017999935?mt=8, consulté le 8 mai 2018.

\section{RÉSUMÉS}

L'apparition des écrans mobiles a engendré un nouveau type de poésie numérique d'ordre tactile. Les poètes numériques expérimentent grâce à ce dispositif une nouvelle manière de produire les effets, les figures et la signification poétiques, renouvelant ainsi l'expérience esthétique ou esthésique du poème sous forme d'application pour smartphones et tablettes tactiles (systèmes iOS et Android). Cet article propose d'envisager, à la suite du «tournant pictural» de la littérature, un devenir tactile de la poésie, caractérisé par un dispositif poétique numérique tactilehaptique.

The recent explosion of mobile devices and touch screens generates a new type of digital poetry, a tactile one. Digital poets experiment through haptic devices a new way of producing poetic effects, tropes and signification, renewing the aesthetic or esthesic experience of a poem as a mobile application for smartphones and touch screens (iOS and Android systems). This article proposes to consider-in the wake of the "pictorial turn" of literature-a becoming tactile of poetry, characterised by a digital haptic-tactile poetic device.

\section{INDEX}

Keywords : digital poetry, mobile device, touch screen, device, poetic device, rhythm, body, sense, sensory experience

Mots-clés : poésie numérique, écran tactile mobile, dispositif, dispositif poétique, rythme, corps, sens, expérience sensorielle

\section{AUTEUR}

\section{EMMANUELLE PELARD}

Université du Luxembourg, IPSE 